\title{
Heterosis Studies for Enhancing Yield and its Attributes in Grasspea (Lathyrus sativus L.)
}

\author{
Amrita Giri*, Abhinao Sao, Deepak Gauraha and H.C. Nanda \\ Department of Genetics and Plant Breeding, Indira Gandhi Krishi Vishwavidalaya, \\ Raipur (CG), India \\ *Corresponding author
}

\section{A B S T R A C T}

Keywords

Grass pea,

Heterosis,

Heterobeltiosis, Standard heterosis, Hybrids

\section{Article Info}

Accepted:

17 October 2019

Available Online:

10 November 2019
Ten $\mathrm{F}_{1}$ hybrids of grass pea and their 5 parents were evaluated in a randomized complete block design in three replication to assess the extent of average heterosis, heterobeltiosis and economic heterosis over standard checks i.e. Mahateora and Prateek for yield and its components. $F_{1}$ were generated in Half diallel mating design. Heterosis for seed yield plant $^{-1}$ was reflected through heterosis in number of branches per plant, number of pods per plant, number of seeds per pod, number of seeds per plant, plant biomass, seed density and seed index. The hybrid Prateek x Siraha Local expressed the highest average heterosis for seed yield per plant followed by Siraha Local x RLS 3004, Ratan x Siraha Local. Similarly, for heterobeltiosis, the highest value was reported by Prateek x Siraha Local followed by Siraha Local x RLS 3004 and heterosis over standard parent was manifested by only one cross Prateek x Siraha Local. Based on per se performance, heterosis and half diallel analysis, the cross combinations Prateek x Siraha Local, Siraha Local x RLS 3004 and Ratan x Siraha Local were found promosing. On the basis of yield these further can be utilized for selection of superior transgressive segregants.

\section{Introduction}

In modern era where burgeoning population is the major problem and increasing global demand for food and feed resources need is a big challenge. In response to fulfillment of growing demands and to need to diversify modern cropping systems (Makoi and Ndakidemi, 2011), the legume genus Lathyrus is receiving increased attention by scientists due to great taste and high nutritional value of the seeds together with cheaper growing requirements.

Grass pea (Lathyrus sativus L.) is an annual pulse crop belonging to the Fabaceae family and Vicieae tribe (Biswas and Biswas, 1997). Grass pea is biotic and abiotic stress resistance 
crop. Compared to other legumes, it is resistant to many insect pests and resistance for changing climatic condition (Tiwari and Campbell, 1996a; Tiwari and Campbell, 1996b; Berger et al., 1999; Sillero et al., 2005). Grass pea is able to withstand in both drought and flooding condition, with wide range of adaptation of soil types, including the marginal ones, to grow in cool climates and at high altitudes. Nowadays, grass pea is grown for stock-feed and human consumption in Asia Middle East (Campbell, 1997), in Northern Africa (Girma et al., 2011) and in Southern Europe (Campbell et al., 1994; Piergiovanni et al., 2011). In fact, these seeds have good protein content (relatively rich in lysine) and a high level of polyunsaturated fatty acids (Chinnasamy et al., 2005) with $41 \%$ carbohydrate, $17 \%$ total dietary fiber (2\% soluble and $15 \%$ insoluble), $2 \%$ fat and $2 \%$ ash, on a dry matter basis but one major drawback which overcome on all advantages is the presence of antinutritional factors (ANFs) in seeds and leaves of grass pea.

In particular, $\beta$-diamino-propionic acid $(\beta$ ODAP), which is a neurotoxic secondary metabolite, is a nonprotein amino acid, which causes neurolathyrism however the levels of the proteinaceous ANFs and of $\beta$-ODAP as well can be reduced by cooking (Enneking, 2011).

\section{Materials and Methods}

The experiment was conducted at Instructional Farm of Indira Gandhi Krishi Vishwavidyalaya, Raipur during the Rabi December 2017-2018. Five diverse genotypes of grass pea namely Mahateora, Ratan, Prateek, Siraha Local, RLS 3004 was chosen and crossed in a half diallel fashion to raise a set of $10 \mathrm{~F}_{1}$ crosses evaluated in a randomized complete block design (RCBD) with three replication. Each replication consisted of fifteen row of two meter length per genotype.
The plant to plant and row to row spacing was $5-7$ and $30 \mathrm{~cm}$, respectively. The observations were recorded from randomly selected five plants on different nineteen traits. The Heterosis was estimated following Fonseca and Patterson (1968).

\section{Results and Discussion}

Investigations on heterosis provides fundamental information regarding the utility of the cross combinations and its use for commercial exploitation. It is estimated for all the nineteen characters. Here we discuss about yield and yield contributing characters only viz. number of primary and secondary branches per plant, number of seeds per pod, number of pods per plant, number of seeds per plant, plant biomass, harvest index, seed index and seed density and among all the crosses attempted in a half diallel cross in grass pea all the three types of heterosis viz. mid-parent heterosis, better- parent heterosis and standard heterosis were estimated.

The value of mid-parent heterosis for number of secondary branches per plant ranged from the value of 9.68 per cent to 15.82 per cent, the cross Mahateora $x$ Ratan expressed the highest significant positive mid parent heterosis, followed by Mahateora x Siraha Local, Ratan x Prateek and Ratan x RLS 3004 (9.68), crosses Mahateora x Ratan, Ratan x RLS 3004 and Mahateora x Siraha Local (15.82) crosses showed significant positive standard heterosis over standard variety Prateek for this trait. For nodes on main stem and number of seeds per pod, the hybrid Prateek x Siraha Local was recorded as the best hybrid as it showed significant positive average heterosis, heterobeltiosis and standard heterosis. For number of pods per plant hybrids, namely Mahateora x Ratan, Ratan x Prateek, Prateek x Siraha Local Ratan x RLS3004 and Mahateora x Siraha Local was found good (Table 1). 


\section{Int.J.Curr.Microbiol.App.Sci (2019) 8(11): 1898-1904}

Table.1 Estimates of mid parent, better parent and standard parent (SH) heterosis for seed yield, its components and (ODAP) content in grass pea

\begin{tabular}{|c|c|c|c|c|c|c|c|c|c|c|c|c|c|c|c|c|c|c|}
\hline \multirow[b]{2}{*}{ HYBRIDS } & \multicolumn{3}{|c|}{ Days to $50 \%$ flowering } & \multicolumn{3}{|c|}{ Days to maturity } & \multicolumn{3}{|c|}{ Plant height } & \multicolumn{3}{|c|}{ Primary branches per plant } & \multicolumn{3}{|c|}{ Sec. branches per plant } & \multicolumn{3}{|c|}{ Node on main stem } \\
\hline & MP & $\mathrm{BP}$ & $\mathrm{BC}$ & MP & $\mathrm{BP}$ & $\mathrm{BC}$ & MP & $\mathrm{BP}$ & $\mathrm{BC}$ & MP & $\mathrm{BP}$ & $\mathrm{BC}$ & MP & $\mathrm{BP}$ & $\mathrm{BC}$ & MP & $\mathrm{BP}$ & $\mathrm{BC}$ \\
\hline 1 & 1.6 & -3.73 & $7.56^{* *}$ & 1.55 & $-3.62 * *$ & 7.31 & 0.86 & $-10.74 * *$ & $-10.74 * *$ & $-32.84 * *$ & $-47.06 * *$ & $-47.06 * *$ & $15.82 * *$ & 8.1 & 8.1 & 9.9 .24 & 5.24 & 13.56 \\
\hline 2 & $7.73 * *$ & 4.81 & $10.82 * *$ & $8.87 * *$ & $4.92 * *$ & 13.12 & $-13.23 * *$ & $-21.22 * *$ & $-21.22 * *$ & $-24.50 * *$ & $-37.65 * *$ & $-37.65 * *$ & 1.48 & -1.9 & -1.9 & -7.69 & -10.16 & -5.08 \\
\hline 3 & -2.18 & $-7.40 * *$ & 3.65 & $5.14 * *$ & 1.59 & 8.95 & $14.83 * *$ & 2.41 & 2.41 & -11.11 & $-27.06^{* *}$ & $-27.06^{* *}$ & $14.01 * *$ & 8.1 & 8.1 & $17.83 * *$ & $16.48^{*}$ & $19.21 * *$ \\
\hline 4 & -0.87 & $-5.13 *$ & 3.78 & $3.88 * *$ & 0 & 8.07 & 5.68 & -4.97 & -4.97 & $-29.17 * *$ & $-40.00^{* *}$ & $-40.00 * *$ & -5.8 & -8.14 & -3.33 & -4.88 & $-12.74 *$ & 4.52 \\
\hline 5 & 2.16 & -0.58 & $11.08 * *$ & $-6.81 * *$ & $-8.29 * *$ & 2.12 & $17.83 * *$ & $14.51 * *$ & -6.57 & 14.94 & 8.3 & $-29.41 * *$ & $13.76 * *$ & 9.69 & 2.38 & 2.12 & 1.05 & 9.04 \\
\hline 6 & $-4.98 * *$ & $-5.07 *$ & $6.26 *$ & -0.94 & $-2.76^{*}$ & 8.27 & -1.89 & -2.74 & $-23.78 * *$ & 19.81 & 13.76 & $-27.06^{* *}$ & 8.59 & 6.8 & -4.29 & 7.48 & 4.71 & 12.99 \\
\hline 7 & $-8.25^{* *}$ & $-9.22 * *$ & 1.43 & $-2.12 *$ & $-3.56 * *$ & 7.38 & $10.41 *$ & 8.43 & $-13.42 * *$ & -3.7 & -11.86 & $-38.82 * *$ & $9.68 *$ & 0 & 5.24 & 1.24 & -3.77 & $15.25^{*}$ \\
\hline 8 & -1.17 & -3.9 & $7.56^{* *}$ & $4.88 * *$ & $4.60 * *$ & 12.78 & $9.60 *$ & 7.44 & $-12.35 * *$ & 7.37 & 6.5 & $-30.59 * *$ & 5.15 & 3.06 & -3.81 & $23.86^{* *}$ & $21.93 * *$ & $28.81 * *$ \\
\hline 9 & -1.45 & -3.1 & $6.00^{* *}$ & $4.17 * *$ & $4.05 * *$ & 12.44 & $9.65^{*}$ & 8.48 & $-11.49 * *$ & 4.9 & 1.69 & $-29.41 * *$ & -8.39 & $-13.57 * *$ & -9.05 & -0.75 & -6.6 & 11.86 \\
\hline 10 & $12.99 * *$ & $11.71^{*}$ & $25.03 * *$ & 0.96 & 0.58 & 8.69 & $10.15^{*}$ & 9.12 & $-12.86^{* *}$ & 20.97 & 16.36 & $-19.24 *$ & -3.64 & $-10.79 *$ & -6.12 & 11.56 & -5.83 & 112.8 \\
\hline
\end{tabular}

*Significant@5\%

**Significant@1\% 


\section{Int.J.Curr.Microbiol.App.Sci (2019) 8(11): 1898-1904}

\begin{tabular}{|c|c|c|c|c|c|c|c|c|c|c|c|c|c|c|c|c|c|c|}
\hline \multicolumn{2}{|c|}{ Hybrids } & \multicolumn{2}{|c|}{ Internodal length } & \multicolumn{3}{|c|}{ Pod per plant } & \multicolumn{3}{|c|}{ Pod length } & \multicolumn{3}{|c|}{ Seed per pod } & \multicolumn{3}{|c|}{ Seed per plant } & \multicolumn{3}{|c|}{ Plant Biomass per plant } \\
\hline & MP & $\mathrm{BP}$ & $\mathrm{BC}$ & MP & $\mathrm{BP}$ & $\mathrm{BC}$ & MP & BP & $\mathrm{BC}$ & MP & $\mathrm{BP}$ & $\mathrm{BC}$ & MP & BP & $\mathrm{BC}$ & MP & BP & $\mathrm{BC}$ \\
\hline 1. & 0.49 & -6.4 & -6.4 & $37.90 * *$ & 4.3 & 4.3 & 10.16 & 2.52 & 2.52 & 12.82 & 10 & 10 & $26.90 * *$ & 0 & 0 & $18.16^{*}$ & 15.13 & $21.35^{*}$ \\
\hline 2. & -3.19 & -8.54 & -8.54 & 3.06 & 2.05 & 4.08 & 7.29 & 6.72 & 6.72 & -2.5 & -2.5 & -2.5 & 3.96 & -1.73 & -1.73 & -0.3 & -12.18 & 15.29 \\
\hline 3. & -5.9 & -10.67 & -10.67 & $15.22 * *$ & $14.77 * *$ & $14.77 * *$ & 0.75 & -1.68 & -1.68 & $-21.61 * *$ & $-36.53 * *$ & 2.5 & 0.25 & -1.49 & -1.49 & $20.93 *$ & 15.56 & 15.56 \\
\hline 4. & 2.11 & 0.74 & 3.51 & 2.79 & -1.61 & 7.61 & $-18.11 * *$ & $-26.90 * *$ & -6.93 & 0 & -4.55 & 5 & 0 & -1.73 & -1.73 & $40.03 * *$ & 14.17 & 14.17 \\
\hline 5. & 10.4 & 8.74 & -3.28 & $45.04 * *$ & 8.97 & $11.14^{*}$ & 6.24 & -0.64 & -1.68 & 5.13 & 2.5 & 2.5 & $36.05 * *$ & $12.03 *$ & -0.25 & -6.59 & $-15.79 *$ & 10.54 \\
\hline 6. & $14.76 * *$ & 12.47 & 1.07 & $34.36 * *$ & 1.89 & 1.1 & $13.32 * *$ & 7.95 & 2.73 & $-35.58 * *$ & $-48.84 * *$ & $-17.38^{*}$ & $28.15 * *$ & 2.31 & -1.24 & 11.94 & 4.36 & 10 \\
\hline 7. & 1.45 & -6.68 & -4.12 & $65.96 * *$ & $21.88 * *$ & $33.30 * *$ & -8.47 & $-23.27 * *$ & -2.31 & -4.88 & -11.36 & -2.5 & $103.4 * *$ & $62.41 * *$ & $56.78 * *$ & $28.89 * *$ & 3.12 & 8.57 \\
\hline 8. & 2.3 & 1.78 & -8.54 & $35.56 * *$ & $33.73 * *$ & $36.38 * *$ & 8.23 & 6.16 & 5.04 & $17.88 * *$ & -4.57 & $54.13 * *$ & $69.18 * *$ & $62.62 * *$ & $56.98 * *$ & $37.03 * *$ & $16.07 *$ & $52.37 * *$ \\
\hline 9. & $15.31 * *$ & 7.57 & 10.52 & -2.87 & -6.15 & 2.65 & $-16.62 * *$ & $-25.91 * *$ & -5.67 & -4.76 & -9.09 & 0 & 3.24 & -0.77 & -4.21 & 17.21 & -13.24 & 13.9 \\
\hline 10. & 4.87 & -1.71 & 0.99 & -4.67 & -9.09 & -0.57 & -4.25 & $-16.34 * *$ & 6.51 & $-19.24 * *$ & $-32.12 * *$ & 9.63 & 0 & 0 & -3.47 & $44.61 * *$ & 22.35 & 11.49 \\
\hline
\end{tabular}

\footnotetext{
*Significant@5\%
}

**Significant@1\% 


\section{Int.J.Curr.Microbiol.App.Sci (2019) 8(11): 1898-1904}

\begin{tabular}{|c|c|c|c|c|c|c|c|c|c|c|c|c|c|c|c|c|c|c|c|c|c|}
\hline & \multicolumn{3}{|c|}{ Seed yield per plant } & \multicolumn{3}{|c|}{ Seed index(gm) } & \multicolumn{3}{|c|}{ Harvest index(\%) } & \multicolumn{3}{|c|}{ ODAP content in flour } & \multicolumn{3}{|c|}{ Seed density(gm) } & \multicolumn{3}{|c|}{ Protein content (\%) } & \multicolumn{3}{|c|}{$\begin{array}{c}\text { ODAP content in seed (\%) } \\
\text {.. }\end{array}$} \\
\hline & MP & BP & $\mathrm{BC}$ & MP & BP & $\mathrm{BC}$ & MP & BP & $\mathrm{BC}$ & MP & BP & $\mathrm{BC}$ & MP & BP & $\mathrm{BC}$ & MP & BP & $\mathrm{BC}$ & MP & BP & $\mathrm{BC}$ \\
\hline 1. & 1.98 & -2.27 & -2.27 & 4.02 & 2.98 & 2.98 & $-14.37^{* *}$ & $-18.76 * *$ & $-18.76 * *$ & 13.04 & 1.96 & -3.70 & -3.13 & $-7.92 * *$ & $-7.92 * *$ & -6.34 & -9.36 & -3.13 & 16.67 & 16.67 & -46.20 \\
\hline 2. & -3.59 & -7.88 & -7.88 & 2.04 & 0 & 4.17 & -4.41 & -6.34 & -6.34 & 2.08 & -3.92 & -9.26 & -0.71 & -3.14 & $-8.25 * *$ & 1.1 & -1.14 & 3.44 & 7.85 & 0.14 & -46.12 \\
\hline 3. & 17.38 & -2.27 & -2.27 & $14.33^{* *}$ & $9.05^{*}$ & $9.05^{*}$ & $-19.08^{* *}$ & $-22.82 * *$ & $-14.96 * *$ & -6.67 & -9.26 & -9.26 & -2.33 & $-4.23 *$ & $-10.23 * *$ & 7.65 & 7.49 & 7.81 & 6.53 & -11.22 & -38.59 \\
\hline 4. & 14.1 & 4.55 & 4.55 & -3.02 & $-7.57 *$ & 1.99 & $-13.26^{* *}$ & $-17.87 * *$ & -8.11 & -14 & -15.69 & $-20.37 * *$ & $4.52^{* *}$ & 3.21 & $-4.62 * *$ & 1.83 & -0.3 & 4.06 & $-57.92 * *$ & $-69.25 * *$ & -69.25 \\
\hline 5. & 9.68 & 9.34 & 0.23 & 0.59 & -2.39 & 1.69 & -1.05 & -4.26 & -8.11 & 13.95 & 8.89 & -9.26 & $-4.07 * *$ & $-6.60 * *$ & $-6.60 * *$ & -1.89 & -2.92 & 3.75 & -7.69 & -14.29 & -53.88 \\
\hline 6. & $26.53 * *$ & 9.17 & 0.08 & $14.90^{* *}$ & $10.65^{* *}$ & $8.45^{*}$ & $-10.24 *$ & $-18.57 * *$ & -10.27 & 13.68 & 0 & 0.00 & $-5.62 * *$ & $-8.58 * *$ & $-8.58 * *$ & 2.87 & -0.29 & 6.56 & -20 & $-33.33 * *$ & -53.88 \\
\hline 7. & $23.26 * *$ & 17.6 & 7.8 & -0.05 & -5.63 & 4.13 & -2.06 & $-11.75^{* *}$ & -1.27 & $-31.11 * *$ & $-36.73 * *$ & $-42.59 * *$ & $-4.63 * *$ & $-8.25 * *$ & $-8.25 * *$ & 5.62 & 4.39 & $11.56^{*}$ & $-57.81 * *$ & $-69.18 * *$ & -69.18 \\
\hline 8. & $93.51 * *$ & $67.40^{* *}$ & $52.50^{* * *}$ & $10.35^{* *}$ & 3.24 & 7.55 & $-18.12^{* *}$ & $-23.40^{* *}$ & $-15.59 * *$ & 3.03 & -5.56 & -5.56 & $-6.13 * *$ & $-6.62 * *$ & $-11.55^{* *}$ & $22.73 * *$ & $20.19 * *$ & $25.75^{* *}$ & -12.37 & -22.11 & -46.12 \\
\hline 9. & 15.58 & 10.6 & 0.76 & 5.38 & 2.43 & $13.02 * *$ & $-14.39 * *$ & $-20.47 * *$ & $-11.03^{*}$ & -8.51 & -12.24 & $-20.37 * *$ & $7.58^{* *}$ & $6.27 * *$ & 0.66 & -1.91 & -2.03 & 2.5 & $-40.03 * *$ & $-53.88^{* *}$ & -53.88 \\
\hline 10. & $36.42 * *$ & $22.70^{*}$ & 2.16 & $8.65^{* *}$ & -0.99 & $9.24 *$ & $-17.09^{*}$ & $-17.71^{* *}$ & -7.94 & $43.69^{* *}$ & $37.04 * *$ & $37.04 * *$ & 3.19 & 2.46 & $-3.96^{*}$ & $-13.17 * *$ & $-14.87 * *$ & $-11.14 *$ & -9.04 & $-23.06^{* *}$ & -23.06 \\
\hline
\end{tabular}

\begin{tabular}{|c|c|c|c|c|c|}
\hline Crosses & 1.Mahateora x.Ratan & 2.Mahateora x Prateek & 3. Mahateora x Siraha local & 4. Mahateora x RLS 3004 & 5. Ratan x Prateek \\
\hline & 6. Ratan x Siraha local & 7. Ratan x RLS 3004 & 8. Prateek x Siraha local & 9. Prateek x RLS 3004 & 10. Siraha local x RLS 3004 \\
\hline MP- Mid parent & BP- Better parent & BC- Best Check & & \\
\hline
\end{tabular}


Similar result was reported by Dahiya (1986), Mourya (1998), Kumari and Prasad (2003) Nanda et al., (2009), Tikariha (2012), Ambade (2013) and Das (2015) for number of pods per plant.

The hybrids Ratan x RLS 3004, Prateek x Siraha Local and Ratan $x$ Prateek was found promising to explot hybrid vigour for number of seeds per plant. Hybrids Ratan x Siraha Local and Mahateora x Siraha Local appeared as the best for seed index, as they found with significant positive average heterosis, heterobeltiosis and standard heterosis over parents and standard checks. Similarly, Prateek x RLS 3004 was investigated as the best for seed density. The value of mid-parent heterosis for seed yield per plant ranged from 23.26 to 93.51. The hybrids Prateek x Siraha Local, Siraha Local x RLS 3004, Ratan x RLS 3004 and Ratan x Siraha Local were observed with significant positive average heterosis over mid parent. The significant positive better-parent heterosis for this trait was recorded only by two hybrids Prateek x Siraha Local and Siraha Local x RLS 3004. Out of ten cross only one Prateek x Siraha Local was recorded with significant positive standard heterosis over both checks. Positive significant heterosis for seed yield per plant was also reported by the researcher's viz. Kumari and Prasad (2003), Nanda et al., (2009), Tikariha (2012) and Das (2015).

\section{References}

Ambade, R.L. (2013). Combining ability studies and molecular characterization in grass pea (Lathyrus sativus L.). M.Sc. Thesis, Deptt. of Plant Breeding and Genetics, IGKV, Raipur, (C.G).

Berger JD, Siddique KHM and Loss SP (1999) Cool season grain legumes for Mediterranean environments: the effects of environment on non-protein amino acids in Vicia and Lathyrus species. Aust J Agr Res 50: 403-412.

Biswas SC, Biswas AK (1997) Induced translocation heterozygosity and sterility in Lathyrus sativus L.. Bangladesh J Botany 26: 131-136.

Campbell, C.G. (1997). Grasspea (Lathyrus sativus $L$.) promoting the conservation and use of underutilized and neglected crops. vol 18. Institute of Plant Genetics and Crop Plant Research, Gatersleben/International Plant Genetic Resources Institute, Rome.

Campbell, C.G., Mehra, R.B., Agrawal S.K., Chen, Y.Z., Abd El Moneim A.M. Khawaja, HIT, Yadov, C.R., Tay, JU and Araya, W.A. (1994). Current status and future strategy in breeding grass pea (Lathyrus sativus). Euphytica 73: 167-175.

Chinnasamy, G., Bal, A.K. and McKenzie, D.B. (2005). Fatty acid composition of grass pea (Lathyrus sativus L.) seeds Lathyrus Lathyrism Newsletter 4: 2-4.

Das, P. 2015. Genetic analysis and molecular characterization in grass pea (Lathyrus sativus L.) M.sc. Thesis, Deptt. of Plant Breeding and Genetics, IGKV, Raipur (C.G.).

Enneking, D. (2011). The nutritive value of grasspea (Lathyrus sativus) and allied species, their toxicity to animals and the role of malnutrition in neurolathyrism. Food. Chem. Toxicol., 49: 694-709.

Girma,A. Tefera, B. and Dadi, L. (2011) Grass pea and neurolathyrism: farmers' perception on its consumption and protective measure in North Shewa, Ethiopia. Food Chem Toxicol, 49 (3): 668-672.

Kumari, V. and Prasad, R. 2003. Model plant type in Khesari (Lathyrus sativus L.) suitable for hill farming. Lathyrus Lathyrism Newsletter, 4: 15-17.

Makoi JHJR, Ndakidemi PA (2011) Changes in plant growth, nutrient dynamics and 
accumulation of flavonoids and anthocyanins by manipulating the cropping systems involving legumes and cereals- a review. Aust. J. Agric. Eng. 2(3): 56-65.

Nanda, H. C., Pandey, R. L., Rao, S. S. and Geda, A.K. 2009. Diallel cross analysis in grass pea (Lathyrus sativus L.). J. Agril. Issues, 13(2): 45-51.

Piergiovanni, A.R., Lupo, F. and Zaccardelli, M. (2011). Environmental effect on yield, composition and technological seed traits of some Italian ecotypes of grass pea (Lathyrus sativus L.). J Sci Food Agric 91 (1): 122129.

Sillero JC, Cubero JI, Fernández-Aparicio M and Rubiales D (2005) Search for resistance to create broomrape
(Orobanche crenata) in Lathyrus. Lathyrus Lathyrism Newsletter 4: 7-9.

Tikariha, K. 2012. Combining ability analysis for yield, its components and neurotoxin (ODAP) content in grass pea (Lathyrus sativus L.). M.Sc. (Ag.) Thesis, Deptt. of Plant Breeding and Genetics, Indira Gandhi Agriculture University, Raipur, India.

Tiwari, K.R. and Campbell, C.G. (1996b) Inheritance of seed weight in grasspea (Lathyrus sativus L.). FABIS Newsletter 38/39: 30-33.

Tiwari, K.R. and Campbell, C.G. (1996a) Inheritance of neurotoxin (ODAP) content, flower and seed coat colour in grass pea (Lathyrus sativus L.). Euphytica 91: 195-203.

\section{How to cite this article:}

Amrita Giri, Abhinao Sao, Deepak Gauraha and Nanda, H.C. 2019. Heterosis Studies for Enhancing Yield and its Attributes in Grasspea (Lathyrus sativus L.). Int.J.Curr.Microbiol.App.Sci. 8(11): 1898-1904. doi: https://doi.org/10.20546/ijcmas.2019.811.222 